\title{
AQP3 small interfering RNA and PLD2 small interfering RNA inhibit the proliferation and promote the apoptosis of squamous cell carcinoma
}

\author{
XIAOYONG WANG, CHENGJUN TAO, CHENGDA YUAN, JINPING REN, MING YANG and HANGYU YING
}

Department of Dermatology, Hangzhou Hospital of Traditional Chinese Medicine, Hangzhou, Zhejiang 310007, P.R. China

Received June 8, 2016; Accepted April 19, 2017

DOI: $10.3892 / \mathrm{mmr} .2017 .6847$

\begin{abstract}
Aquaporin 3 (AQP3) and phospholipase D2 (PLD2) are abnormally expressed and/or localized in squamous cell carcinoma (SCC). AQP3 transports glycerol to PLD2 for the synthesis of lipid second messenger, which can mediate the effect of the AQP3/PLD2 signaling module in the regulation of keratinocyte proliferation and differentiation. However, the role of the AQP3/PLD2 signaling module in the pathogenesis of SCC remains to be fully elucidated. In the present study, the expression levels of AQP3 and PLD2 in tissue samples were examined using immunohistochemistry, it was found that the expression levels of AQP3 and PLD2 in tissue samples of actinic keratosis (AK), Bowen's disease (BD) and SCC were significantly increased. AQP3 small interfering RNA (siRNA) and PLD2 siRNA were constructed and used for transfection into the human A431 SCC cell line, and their anticancer effect on SCC was examined. The mRNA expression and protein expression levels of AQP3 and PLD2 were significantly downregulated following siRNA transfection. AQP3 siRNA and PLD2 siRNA inhibited the proliferation and promoted the apoptosis of A431 cells. Taken together, the findings of the present study suggested that increased levels of AQP3 and PLD2 were correlated with tumor progression and development in SCC. AQP3 siRNA and PLD2 siRNA significantly downregulated the mRNA and protein levels of AQP3 and PLD2 in the A431 cells; inhibiting proliferation and promoting apoptosis in vitro. The concomitant effects of AQP3/PLD2 signaling by inhibiting the expression of siRNA may be important for the treatment of SCC in the future.
\end{abstract}

Correspondence to: Dr Xiaoyong Wang, Department of Dermatology, Hangzhou Hospital of Traditional Chinese Medicine, 453 Tiyuchang Road, Hangzhou, Zhejiang 310007, P.R. China E-mail: wangxiaoyong1974@163.com

Abbreviations: AQP3, aquaporin 3; PLD2, phospholipase D2; SCC, squamous cell carcinoma; AK, actinic keratosis; BD, Bowen's disease; siRNA, small interfering RNA; FITC/PI, annexin V-fluorescein isothiocyanate/propidium iodide

Key words: aquaporin 3, phospholipase D2, squamous cell carcinoma

\section{Introduction}

The aquaporins (AQPs) are a family of membrane proteins, which form water channels across cell membranes. There are 13 AQPs in mammals, named AQP0 to AQP12. AQP 1, 2, 4, 5 and 8 appear to function as selective water channels, whereas AQP 3, 7, 9 and 10 can transport water and glycerol. There are up to six AQPs (AQP 1, 3, 5, 7,9 and 10) expressed in the skin. AQP1 has been detected in melanocytes and endothelial cells of the human dermis (1). Boury-Jamot et al (2) demonstrated that AQP3 was expressed in the plasma membrane of human keratinocytes in vitro and in vivo (2). AQP5 is expressed in sweat glands (3), AQP7 is expressed in adipocytes (4), AQP9 is expressed in preadipocytes and AQP10 is expressed in keratinocytes (2).

AQP3 is the most abundant skin aquaglyceroporin (5). AQP3 is expressed at high levels in keratinocyte plasma membranes of the human epidermis and reconstructed human epidermis (6). AQP3 is involved in the differentiation and proliferation of keratinocytes. Using an RNase protection assay, it has been shown that AQP3 mRNA is expressed in growing and differentiating human keratinocytes (7). AQP3 is overexpressed in human skin squamous cell carcinoma (SCC) (8). AQP3-null mice show considerable resistance to the development of skin tumors following exposure to tumor initiators (8). Therefore, AQP3 may be an important determinant in skin tumorigenesis, and a novel target for tumor prevention and therapy (8).

Phospholipase D (PLD) was first identified in plants as a distinct phospholipid-specific phosphodiesterase, which hydrolyses phosphatidylcholine to phosphatidic acid (PA) and choline (9). PLD is a phospholipid-degrading enzyme, which generates biologically active products that are considered to have important functions in cell regulation (10). The activity of PLD results in modification of various lipid constituents of the membrane, and generates one or more messenger molecules, which are able to recruit or modulate specific target proteins. PA is product of the PLD enzymatic action and is a major lipid second messenger, which regulates signaling pathways and cell proliferation. There are two PLD isoforms in mammals, PLD1 and PLD2. PLD is important in tumorigenesis, and the elevation of either PLD1 or PLD2 contributes to cancer progression. Elevated PLD activity and expression have been reported in several types of cancer (11). PLD provides survival 
signals and is involved in the migration, adhesion and invasion of cancer cells (11).

AQP3 and PLD2 are co-localized in caveolin-rich membrane microdomains of keratinocytes. AQP3 and PLD2 form a signaling module in lipid rafts, where AQP3 transports glycerol to PLD2 for the synthesis of phosphatidylglycerol (PG). PG can mediate the effects of the AQP3/PLD2 signaling module in the regulation of keratinocyte proliferation and differentiation (12). AQP3 and PLD2 are abnormally expressed and/or localized in SCC, basal cell carcinoma and psoriasis, thus the AQP3/PLD2 signaling module may be involved in, or serve as surrogate markers for the pathogenesis of these diseases (13).

To confirm the role of AQP3 and PLD2, and the AQP3/PLD2 signaling module in SCC, the present study examined the protein expression and localization of AQP3 and PLD2 in actinic keratosis (AK), Bowen's disease (BD) and $\mathrm{SCC}$, relative to the normal epidermis. The anticancer effects of AQP3 small interfering RNA (siRNA) and PLD2 siRNA on SCC were also examined.

\section{Materials and methods}

Skin tissue samples. To analyze the expression of AQP3 and PLD2, paraffin-embedded tissue sections of skin from patients with diagnoses of AK, BD and SCC, as determined and verified by two dermatopathologists, were obtained from the tissue archives of the Department of Pathology at Hangzhou Hospital of Traditional Chinese Medicine (Hangzhou, China). Normal skin tissue samples of 10 patients ( 5 females, 5 males) were obtained from skin biopsies from October 1 to 31 2013, the remaining patient details are presented in Table I. The clinical and histopathological characteristics of the patients are shown in Table I. The procedures were approved by the Ethics Committee of Hangzhou Hospital of Traditional Chinese Medicine and informed consent was obtained from the patient.

AQP3 and PLD2 immunohistochemistry. Immunohistochemistry was performed as previously described (14). The slides used for AQP3 staining were deparaffinized, washed twice for $5 \mathrm{~min}$ in phosphate-buffered saline (PBS), incubated for $10 \mathrm{~min}$ at $37^{\circ} \mathrm{C}$ in $3 \%$ hydrogen peroxide, washed three times for $5 \mathrm{~min}$ in PBS, incubated for $30 \mathrm{~min}$ in $0.3 \%$ goat serum (Maixin Biotech Co., Ltd., Fuzhou, China), and then incubated overnight with the following primary antibodies: Rabbit anti-AQP3 (cat. no. BS3671; Bioworld Technology, Inc., St. Louis Park, MN, USA; 1:200 dilution); rabbit-anti-PLD2 (cat. no. AP14669a; Abgent, Inc., San Diego, CA, USA; 1:100 dilution), in a humidified chamber at $4^{\circ} \mathrm{C}$. Following secondary antibody (cat. no. AP40467a; Maixin Biotech Co., Ltd.; 1:100 dilution) incubation for $15 \mathrm{~min}$ at $37^{\circ} \mathrm{C}$, an $\mathrm{ABC}$ staining kit (Santa Cruz Biotechnology, Inc., Santa Cruz, CA, USA) was used to visualize immunoreactivity with Olympus CX21FS1C microscope and development with the chromogen 3,30-diaminobenzidine (Maixin Biotech Co., Ltd.) for $3 \mathrm{~min}$.

The expression levels of AQP3 and PLD2 on each slide were graded according to a previously described scoring system (14). Each slide was scored according to staining intensity and the proportion of positive cells. The scores of staining intensity were: 0 , negative staining; 1 , mild staining; 2, moderate staining; 3, severe staining. The scores for the proportion of positive cells were: $1,<33 \%$ positive cells; 2 , $33-66 \%$ positive cells; $3,>66 \%$ positive cells. The final score of each slide was the multiplication result of the two above scores. The results of the immunohistochemical staining were graded according to the final score in a semiquantitative manner on a four-point scale:-, negative expression; +, low expression (score 1-3); ++, moderate expression (score 4-6); and +++ , high expression (score 7-9).

Cell culture. The human A431 SCC cell line was purchased from the China Center for Type Culture Collection (Wuhan, China). The A431 cells were maintained at $37^{\circ} \mathrm{C}$ in a humidified atmosphere $\left(95 \%\right.$ air and $\left.5 \% \mathrm{CO}_{2}\right)$ and grown in plastic tissue-culture flasks containing DMEM (Gibco; Thermo Fisher Scientific, Inc., Waltham, MA, USA) with $10 \%$ fetal bovine serum (Gibco; Thermo Fisher Scientific, Inc.).

Transfection of siRNAs. The three specific siRNAs (AQP3 siRNA, PLD2 siRNA and negative control siRNA) were designed by GenePharma (Shanghai, China). The sequences are shown in Table II. Transfection of the A431 cells with AQP3 siRNA and PLD2 siRNA was performed according to the manufacturer's protocol and as previously described (15). Briefly, 50,000 cells $/ \mathrm{cm}^{2}$ were plated into 6-well plates and allowed to adhere for $24 \mathrm{~h}$. Subsequently, $5 \mu \mathrm{l}$ of siRNA was added to $250 \mu \mathrm{l}$ of Opti-MEM (Gibco; Thermo Fisher Scientific, Inc.) thoroughly mixed, and incubated at room temperature for $5 \mathrm{~min}$. Lipofectamine ${ }^{\mathrm{TM}} 2000(5 \mu \mathrm{l}$; Gibco; Thermo Fisher Scientific, Inc.) was added to $250 \mu 1$ of Opti-MEM, thoroughly mixed and incubated at room temperature for $5 \mathrm{~min}$. The diluted siRNA and diluted Lipofectamine $^{\mathrm{TM}} 2000$ were mixed and incubated at room temperature for $20 \mathrm{~min}$. The siRNA/Lipofectamine mixture was transferred into 6 -well plates at $500 \mu \mathrm{l} /$ well. The cells were maintained for $6 \mathrm{~h}$ at $37^{\circ} \mathrm{C}$. Following replacement of the culture medium, the cells were incubated for an additional 24-72 h. AQP3- and PLD2-knockdown were verified using reverse transcription-quantitative polymerase chain reaction (RT-qPCR) and western blot analyses.

RT- $q$ PCR analysis. Total RNA was extracted from A431 cells using TRIzol (Invitrogen; Thermo Fisher Scientific, Inc.) at $24 \mathrm{~h}$ post-siRNA transfection. cDNA was synthesized from the isolated RNA using a RevertAid First Strand cDNA synthesis kit (Fermentas; Thermo Fisher Scientific, Inc.) according to the manufacturer's protocol.

The qPCR assay was performed on a CFX Connect Real-Time PCR detection system (Bio-Rad Laboratories, Inc., Hercules, CA, USA) according to the manufacturer's protocol. The qPCR analysis was performed with $25 \mu$ ( (final volume) reaction mixture, containing $10.5 \mu \mathrm{l}$ of $\mathrm{cDNA}, 12.5 \mu \mathrm{l}$ of IQ SYBR Green Supermix (Bio-Rad Laboratories, Inc.) and $1 \mu \mathrm{l}$ each of $10 \mu \mathrm{M}$ forward and reverse primers (Takara Bio, Inc., Kusatsu, Otsu, Japan). The following primers were used for amplification of AQP3, PLD2 and the internal control (GAPDH): AQP3, forward 5'-CCCCTCTGGACACTTGGA T-3' and reverse 5'-CACGAAGACACCCGCAAT-3'. PLD2, forward 5'-GCCTTGGGCATCAACAGT-3' and reverse 
5'-AGGTCAGTCAGTCGGTAGTG-3'. GAPDH, forward 5'-AGA AGGCTGGGGCTCATTTG-3' and reverse 5'-AGG GGCCATCCACAGTCTTC-3'. Thermal cycling was initiated with an initial denaturation step at $50^{\circ} \mathrm{C}$ for $3 \mathrm{~min}, 95^{\circ} \mathrm{C}$ for $3 \mathrm{~min}$, followed by 40 cycles of $95^{\circ} \mathrm{C}$ for $10 \mathrm{sec}, 61^{\circ} \mathrm{C}$ for $20 \mathrm{sec}$ and $72^{\circ} \mathrm{C}$ for $20 \mathrm{sec}$. The $2^{-\Delta \Delta \mathrm{Cq}}$ method (16) was used for data analysis, with results representative of three independent experiments.

Western blot analysis. Total protein was extracted using a protein extraction kit (Active Motif, Carlsbad, CA, USA) and protein concentration was determined using the Bradford method (Active Motif) according to manufacturer's protocol at $24 \mathrm{~h}$ following siRNA transfection and western blot analysis was performed as previously described (17) with the following primary and secondary antibodies: Rabbit-anti-AQP3 cat. no. sc-9885; Santa Cruz Biotechnology, Inc.; 1:1,000 dilution), rabbit-anti-PLD2 (cat. no. AP14669a; Abgent, Inc.; 1:1,000 dilution), rabbit-anti-GAPDH (cat. no. AP0063; Bioworld Technology, Inc.; 1:1,000 dilution) and horseradish peroxidase-conjugated goat anti-rabbit IgG (cat. no. BS13278; Bioworld Technology, Inc.; 1:1,000 dilution). Briefly, $25 \mu \mathrm{g}$ of protein was electrophoresed on a $10 \%$ sodium dodecyl sulfate-polyacrylamide gel. The protein was then transferred onto a polyvinylidene fluoride membrane. The membrane was incubated with primary antibody for $2 \mathrm{~h}$ at room temperature and then incubated with secondary antibody for $1 \mathrm{~h}$ at room temperature. The bands were visualized chemiluminescently using an ECL kit (Beyotime Institute of Biotechnology, Shanghai, China). Semi-quantification was verified using Image J version 1.41 software (imagej. nih.gov/ij/), with results representative of three independent experiments.

Cell proliferation assay. A Cell Counting Kit-8 (Dojindo Molecular Technologies, Inc., Kumamoto, Japan) was used to measure the effects of siRNA transfection on the proliferation of A431 cells, according to the manufacturer's protocol. The Cell Counting Kit- 8 assay was performed in 96 -well plates 24, 48 and $72 \mathrm{~h}$ following siRNA transfection. Cells were plated at a density of $5 \times 10^{4}$ cells/well into 96 -well plates. The detection reagent (10 $\mu \mathrm{l}$ WST-8) was added to each well and incubated for $1 \mathrm{~h}$ at $37^{\circ} \mathrm{C}$. Viable cell numbers were estimated by measurement of the optical density at $450 \mathrm{~nm}$, with results representative of six independent experiments.

Cell apoptosis assay. Cell apoptosis was detected using Annexin V-fluorescein isothiocyanate/propidium iodide (FITC/PI) double labeling with an Alexa Fluor 488 Annexin V/Dead Cell Apoptosis kit (Invitrogen; Thermo Fisher Scientific, Inc.) according to the manufacturer's protocol. Cells were plated at $2 \times 10^{5}$ cells/well into 6 -well plates. Annexin V-FITC/PI double labeling was performed in 6-well plates 24, 48 and $72 \mathrm{~h}$ following siRNA transfection. Annexin V-FITC $(5 \mu \mathrm{l})$ was added to each well and incubated for $15 \mathrm{~min}$ at $4^{\circ} \mathrm{C}$, following which $10 \mu \mathrm{l}$ of PI was added and incubated for $5 \mathrm{~min}$ at $4^{\circ} \mathrm{C}$. The stained cells were analyzed using BD FACSVerse flow cytometry (BD Biosciences, San Diego, CA, USA), with results representative of three independent experiments.
Statistical analysis. Data were statistically analyzed using the Statistical Package for Social Sciences, version 12.0 (SPSS, Inc., Chicago, IL, USA). The results are expressed as the mean \pm standard deviation. $\mathrm{P}<0.05$ was considered to indicate a significant difference in all analyses. For the results of the immunohistochemical staining, the statistical significance between two groups was determined using a Mann-Whitney $\mathrm{U}$ test. For other results, the statistical significance between two groups was determined using a paired-samples t-test. The statistical significance between multiple groups was determined using one-way analysis of variance in conjunction with a Newman Keuls post-hoc test.

\section{Results}

Expression of AQP3 is increased in tissue samples of $A K$, $B D$ and $S C C$. The present study examined the expression of AQP3 in tissue samples of normal skin, AK, BD and SCC using immunohistochemistry, as shown in Fig. 1. This analysis revealed low expression levels of AQP3 in normal epidermal keratinocytes and prominent expression in the middle and lower epidermis (Fig. 1A). In the tissue samples of AK and BD, AQP3 was moderately expressed in the epidermis (Fig. 1B and C). In the tissue samples of SCC, AQP3 was moderately expressed in the horn pearls and carcinoma cells nests (Fig. 1D). This analysis also showed prominent expression of AQP3 in the plasma membrane of keratinocytes, consistent with the fact that AQP3 is an integral membrane protein (6). AQP3 was negatively expressed in the dermal tunica intima of the normal skin, AK, BD and SCC samples.

Compared with normal skin, the expression levels of AQP3 in the tissue samples of AK, BD and SCC were significantly increased (z-values of 4.175, 4.369 and 4.818 respectively; $\mathrm{P}<0.01$ ), as shown in Fig. 1E.

Expression of PLD2 is increased in tissue samples of $A K, B D$ and $S C C$. The present study also examined the expression levels of PLD2 in tissue samples of normal skin, AK, BD and SCC using immunohistochemistry, as shown in Fig. 2. This analysis revealed a low level of expression of PLD2 in normal epidermal keratinocytes and prominent expression in the middle and lower epidermis (Fig. 2A). In the tissue samples of AK, PLD2 was moderately expressed in the epidermis (Fig. 2B). In the tissue samples of BD and SCC, PLD2 was expressed at high levels (Fig. 2C and D). The majority of previous investigations have reported that PLD2 localizes to the plasma membrane (18), however, it has also been reported to have a cytosolic distribution and localize to the Golgi apparatus (7). The results of the present study showed that PLD2 localized to the cytoplasm and plasma membrane of keratinocytes.

Compared with normal skin, the expression levels of PLD2 in the tissue samples of AK, BD and SCC were significantly increased (z-value: 4.09, 4.31 and 4.74, respectively; $\mathrm{P}<0.01$ ), as shown in Fig. 2E.

Selection and identification of siRNA. Based on the cDNA sequences of AQP3 and PLD2 in GenBank, three sequences of AQP3 siRNA and three sequences of PLD2 siRNA were designed. The sequences used are shown in Table II. The A431 
Table I. Clinical and histological characteristics of patients and tissue samples.

\begin{tabular}{|c|c|c|c|c|}
\hline Characteristic & Control & Actinic keratosis & Bowen's disease & Squamous cell carcinoma \\
\hline Cases (n) & 10 & 12 & 15 & 20 \\
\hline Age $\left(\right.$ years) ${ }^{\mathrm{a}}$ & $73.43 \pm 8.49$ & $79.86 \pm 8.09$ & $71 \pm 6.51$ & $67.67 \pm 14.04$ \\
\hline \multicolumn{5}{|l|}{ Site (n) } \\
\hline Head & 4 & 9 & 0 & 7 \\
\hline Trunk & 3 & 0 & 9 & 3 \\
\hline Limbs & 3 & 3 & 6 & 10 \\
\hline siRNA & \multicolumn{2}{|c|}{ Sense $\left(5^{\prime}-3^{\prime}\right)$} & \multicolumn{2}{|c|}{ Antisense (5'-3') } \\
\hline \multicolumn{5}{|c|}{ Tumor stage $(n)^{b}$} \\
\hline I & & & & 10 \\
\hline II & & & & 4 \\
\hline III & & & & 4 \\
\hline IV & & & & 2 \\
\hline
\end{tabular}

a Data presented as the mean \pm standard deviation; 'Broders' classification.

Table II. Sequences of siRNA.

\begin{tabular}{lcc}
\hline siRNA & Sense (5'-3') & Antisense (5'-3') \\
\hline AQP3-homo-612 & CCCUUAUCGUGUGUGUGCUTT & AGCACACACACGAUAAGCGTT \\
AQP3-homo-363 & CCUUUGCCAUGUGCUUCCUTT & AGGAAGCACAUGGCAAAGGTT \\
AQP3-homo-360 & GGGCUGUAUUAUGAUGCAATT & UUGCAUCAUAAUACAGCCCTT \\
PLD2-homo-602 & CAGCCAGCAAACAGAAAUATT & UAUUUCUGUUUGCUGGCUGTT \\
PLD2-homo-1352 & GGCAUCAACAGUGGCUAUATT & UAUAGCCACUGUUGAUGCCTT \\
PLD2-homo-5338 & GGCACCGAAAGAUAUACCATT & UGGUAUAUCUUUCGGUGCCTT \\
Negative control & UUCUCCGAACGUGUCACGUTT & ACGUGACACGUUCGGAGAATT \\
\hline
\end{tabular}

siRNA, small interfering RNA; AQP3, aquaporin 3; PLD2, phospholipase 2.

cells were transfected with siRNA using Lipofectamine ${ }^{\mathrm{TM}}$ 2000. The inhibitory effect of each siRNA sequence on the mRNA expression of the target gene was detected using RT-qPCR analysis, as shown in Fig. 3. In the three AQP3 siRNA sequences, AQP3-homo-363 had the most marked inhibitory effect on the mRNA expression of AQP3, however, it also inhibited the mRNA expression of PLD2. Therefore, AQP3-homo-612 was selected for use in the following experiments. In the three PLD2 siRNA sequences, PLD2-homo-602 had the highest inhibitory effect on the mRNA expression of PLD2, therefore, PLD2-homo-602 was selected for use in the following experiments.

The inhibitory effect of AQP3-homo-612 siRNA on the protein expression of AQP3, and the inhibitory effect of PLD2-homo-602 siRNA on the protein expression of PLD2 were detected using western blot analysis, as shown in Fig. 4A. The protein level of AQP3 was significantly downregulated following siRNA transfection, compared with negative control siRNA transfection $(\mathrm{t}=60.884$; $\mathrm{P}<0.001$; Fig. 4B). The protein level of PLD2 was also significantly downregulated following siRNA transfection, compared with negative control siRNA transfection ( $\mathrm{t}=12.419 ; \mathrm{P}=0.006)$ (Fig. 4C). No significant difference was found among the $\mathrm{CK}$ group, transfection regent group and negative control group $(\mathrm{P}>0.05)$. These results indicated that the repression of protein levels of AQP3 and PLD2 occurred post-transcriptionally.

AQP3 siRNA and PLD2 siRNA suppress the proliferation of A431 cells. The present study also determined the proliferation of A431 cells following AQP3 siRNA and PLD2 siRNA transfection. As shown in Fig. 5, compared with the negative control group, the proliferation of A431 cells following AQP3 siRNA transfection was significantly inhibited at 24, 48 and $72 \mathrm{~h}$ $(\mathrm{t}=10.997,24.103$ and 9.54, respectively; $\mathrm{P}<0.01)$. Compared with the negative control group, the proliferation of A431 cells following PLD2 siRNA transfection was also significantly inhibited at 24, 48 and $72 \mathrm{~h}(\mathrm{t}=8.726,30.468$ and 7.017, respectively; $\mathrm{P}<0.01)$. Compared with the negative control group, the proliferation of A431 cells following AQP3 siRNA and PLD2 siRNA transfection was also significantly inhibited at 24, 48 and $72 \mathrm{~h}(\mathrm{t}=12.496,34.732$ and 11.459, respectively; $\mathrm{P}<0.01)$. No significant differences were found among the CK group, transfection reagent group and negative control group $(\mathrm{P}>0.05)$.

AQP3 siRNA and PLD2 siRNA promote the apoptosis of A431 cells. The apoptosis of A431 cells following transfection with 
A

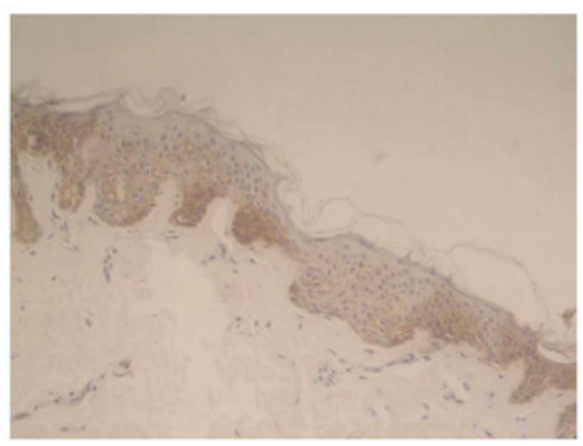

C

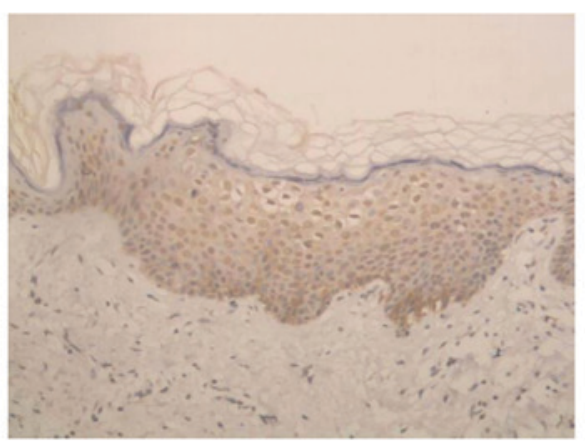

B

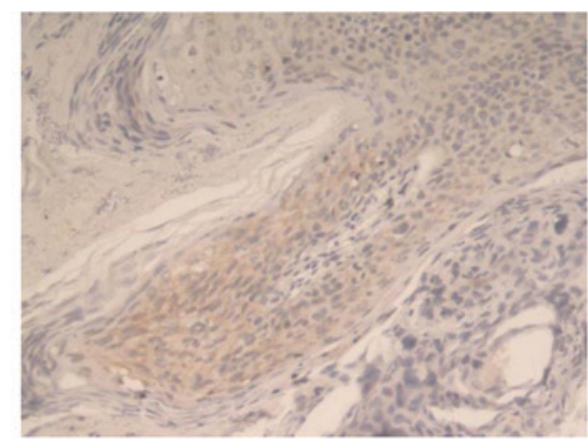

D

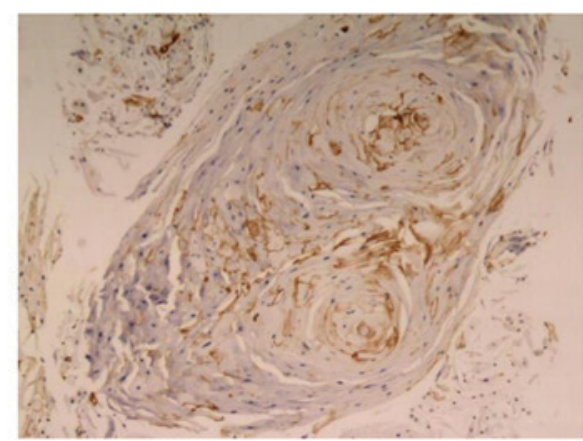

$E$

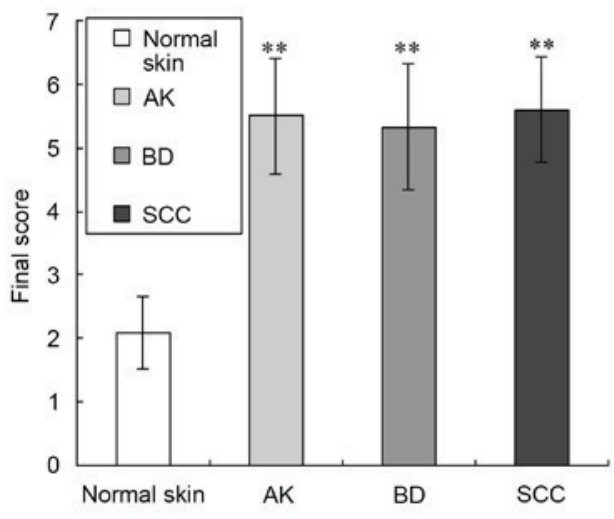

Figure 1. Expression of AQP3 is increased in tissue samples of AK, BD and SCC Magnification, $x 200$. Skin tissue samples were obtained and AQP3 immunohistochemistry was performed. (A) Expression of AQP3 in the tissue samples of normal skin. (B) Expression of AQP3 in tissue samples of AK. (C) Expression of AQP3 in tissue samples of BD. (D) Expression of AQP3 in tissue samples of SCC. (E) Differences in the expression of AQP3 between normal skin and AK, $\mathrm{BD}, \mathrm{SCC}$. Results are expressed as the mean \pm standard deviation. Statistical significance between two groups was determined using a Mann-Whitney U test. ${ }^{* *} \mathrm{P}<0.01$, compared with normal skin. AQP3, aquaporin 3; AK, actinic keratosis; $\mathrm{BD}$, Bowen's disease; SCC, squamous cell carcinoma.

AQP3 siRNA and PLD2 siRNA were also determined. As shown in Fig. 6, compared with the negative control group, the apoptosis of A431 cells following AQP3 siRNA transfection was significantly increased at 48 and $72 \mathrm{~h}(\mathrm{t}=11.359$ and 20.912 , respectively; $\mathrm{P}<0.01$ ). Compared with the negative control group, the apoptosis of A431 cells following PLD2 siRNA transfection was significantly increased at $72 \mathrm{~h}$ $(\mathrm{t}=19.891 ; \mathrm{P}<0.01)$. Compared with the negative control group, the apoptosis of A431 cells following AQP3 siRNA and PLD2 siRNA transfection was significantly increased at 48 and $72 \mathrm{~h}$ $(\mathrm{t}=15.559$ and 27.692, respectively; $\mathrm{P}<0.01)$. No significant differences were found among the CK group, transfection regent group and negative control group $(\mathrm{P}>0.05)$.

\section{Discussion}

Hara-Chikuma and Verkman demonstrated AQP3 immunoreactivity in human SCC (8). It was suggested that the protein expression of AQP3 is correlated with proliferation in SCC as the channel is co-localized with keratin 14, a marker of basal keratinocytes (8). Verkman et al (19) also indicated that the levels of AQP3 were increased in SCC and correlated with the hyperproliferation observed in the disease (19). However, Voss et al (13) examined AQP3 immunoreactivity in SCC and found that AQP3 immunoreactivity was 'patchy', with certain regions of the lesion staining intensely for AQP3 and others showing minimal or no staining. The 
A

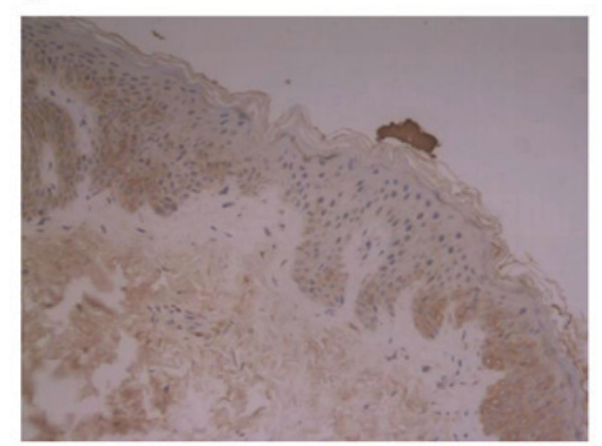

C

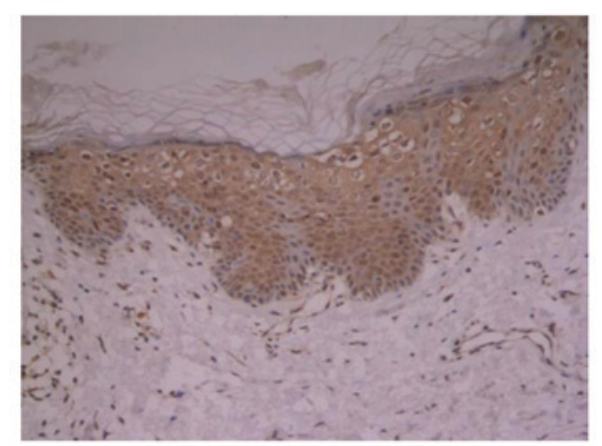

B

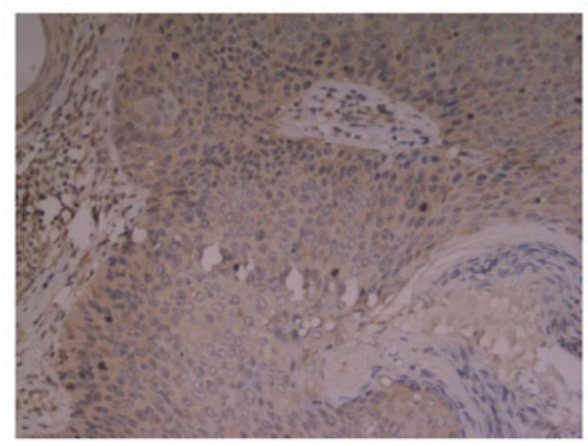

D

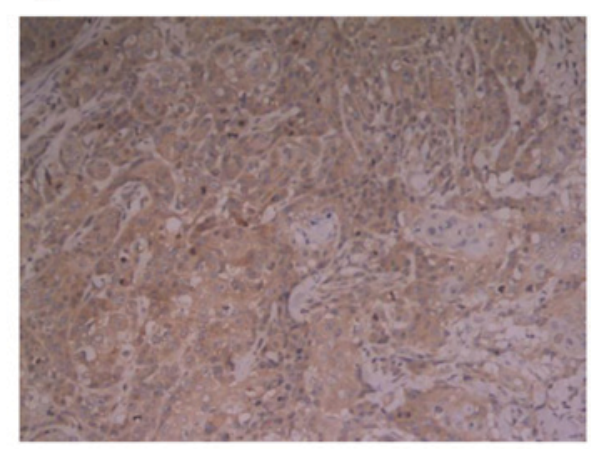

$\mathrm{E}$

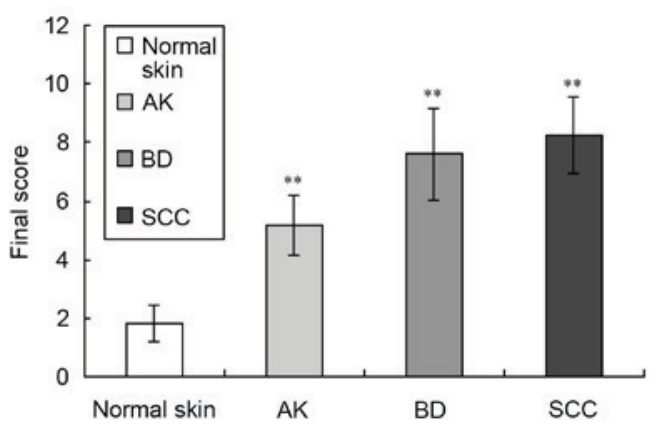

Figure 2. Expression of PLD2 is increased in tissue samples of AK, BD and SCC. Magnification, x200. Skin tissue samples were obtained and PLD2 immunohistochemistry was performed. (A) Expression of PLD2 in tissue samples of normal skin. (B) Expression of PLD2 in tissue samples of AK. (C) Expression of PLD2 in tissue samples of BD. (D) Expression of PLD2 in SCC. (E) Differences in the expression of PLD2 between normal skin and AK, BD, SCC samples. Results are expressed as the mean \pm standard deviation. Statistical significance between two groups was determined using a Mann-Whitney U test. ${ }^{* *} \mathrm{P}<0.01$, compared with normal skin. PLD2, phospholipase D2; AK, actinic keratosis; BD, Bowen's disease; SCC, squamous cell carcinoma.

regions showing reduced levels of AQP3 exhibited positivity for Ki67, a marker of proliferating cells, whereas regions of the tumor with high levels of AQP3 were negative for Ki67 immunoreactivity (13). This suggested that proliferation is correlated with the downregulation of AQP3 in SCC (13). In the present study, the levels of AQP3 were determined in $\mathrm{AK}, \mathrm{BD}$ and $\mathrm{SCC}$ tissues using immunohistochemistry, and compared with the normal epidermis. It was found that the expression of AQP3 was low in the normal epidermis, whereas $\mathrm{AQP} 3$ was moderately expressed in $\mathrm{AK}$ and $\mathrm{BD}$, and moderately expressed in the horn pearls and carcinoma cells nests of SCC. Compared with normal skin, the expression levels of AQP3 in AK, BD and SCC tissues were significantly increased. $\mathrm{AK}$ is an intra-epidermal keratinocyte-derived precancerous lesion in humans. BD is a variant of SCC in the skin and the mucocutaneous junction (20). The findings of the present study suggested that increased levels of AQP3 were correlated with proliferation in SCC, and with tumor progression from a precancerous lesion to a cancerous lesion in situ, and then to an invasive lesion.

The role of PLD in cancer and tumorigenesis has been investigated in detail in the last decade. The activity of PLD has been shown to be significantly elevated in several types of tumor (11), suggesting the possibility that PLD may be involved in tumorigenesis. The overexpression of PLD1 and PLD2 has been found to induce upregulation of the activity of matrix metalloprotease-9, induce undifferentiated sarcoma when transplanted into nude mice and increase the fraction of cells in the S phase. These results suggest that overexpression of PLD isozymes may be important in neoplastic transformation (21). A431 cells express PLD1 and PLD2, however, the regulatory mechanism through which PLD1 and 


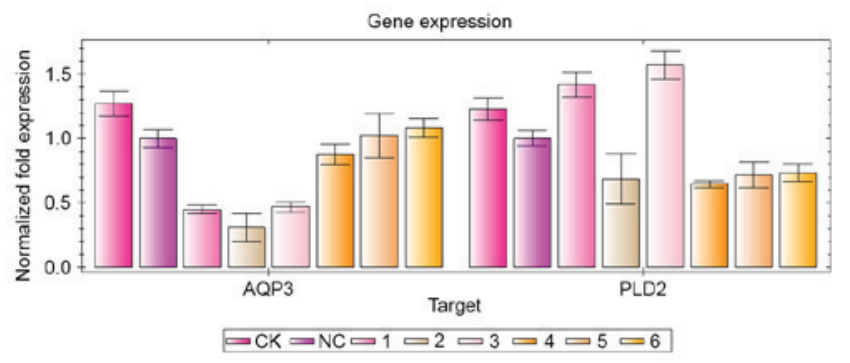

Figure 3. Inhibitory effects of AQP3 and PLD2 siRNA sequences on the target gene. siRNA transfection and reverse transcription-quantitative polymerase chain reaction analyses were performed. The groups were as follows: $\mathrm{CK}$ group, normal cultured A431 cells; NC group, A431 cells were transfected with NC siRNA; 1, AQP3-homo-612 siRNA group; 2, AQP3-homo-363 siRNA group; 3, AQP3-homo-360 group; 4, PLD2-homo-602 group; 5, PLD2-homo-1352 group; 6, PLD2-homo-5338 group. siRNA, small interfering RNA; AQP3, aquaporin 3; PLD2, phospholipase D2; CK, control check; NC, negative control.

PLD2 are activated is different. Hydrogen peroxide induces the tyrosine phosphorylation of PLD1 and PLD2, whereas epidermal growth factor only causes the tyrosine phosphorylation of PLD2 (22). The involvement of PLD2 in cell signaling continues to expand geometrically. It involves gene transcription, and mitogenic and cell migration effects as observed in normal growth, tumor development and inflammation (23). The present study also determined the levels of PLD2 in AK, BD and SCC using immunohistochemistry. It was found that the expression of PLD2 was low in the normal epidermis, whereas PLD2 was moderately expressed in AK and expressed at high levels in BD and SCC. Compared with normal skin, the expression levels of PLD2 in the tissue samples of AK, BD and SCC were significantly increased. These findings suggested that increased levels of PLD2 may be correlated with tumor progression and development in SCC.

On investigating gene function, the specific knockdown of target genes without affecting other genes is critically important. RNA interference mediated by siRNA and short hairpin RNA is a specific gene-silencing technology. Based on the AQP3 and PLD2 cDNA sequences in GenBank, three specific sequences of AQP3 siRNA and three specific sequences of PLD2 siRNA were constructed in the present study for transfection into the human A431 SCC cell line. Different siRNAs had different inhibitory effects on the mRNA expression of the target gene. The positional effects and a variance in the secondary structure of the nucleotide sequence at different sites are involved in the different inhibitory effects of the siRNA targeting the same gene $(24,25)$. The ability to evade apoptosis is a hallmark of cancer cells, resulting in tumor growth, metastasis, and resistance to chemotherapy and radiotherapy. Using in vitro experiments, the present study confirmed the anticancer effect of AQP3 siRNA and PLD2 siRNA on SCC. The results showed that AQP3 siRNA and PLD2 siRNA affected the proliferation and apoptosis of SCC. Transfection with AQP3 siRNA and PLD2 siRNA significantly inhibited proliferation and promoted apoptosis of SCC cells. Several investigations have shown that silencing PLD has a negative effect on the migration, adhesion and invasion of cancer cells. The concomitant downregulation of siRNA mediated by aberrant AQP3/PLD2 signaling may be important for the treatment of SCC.
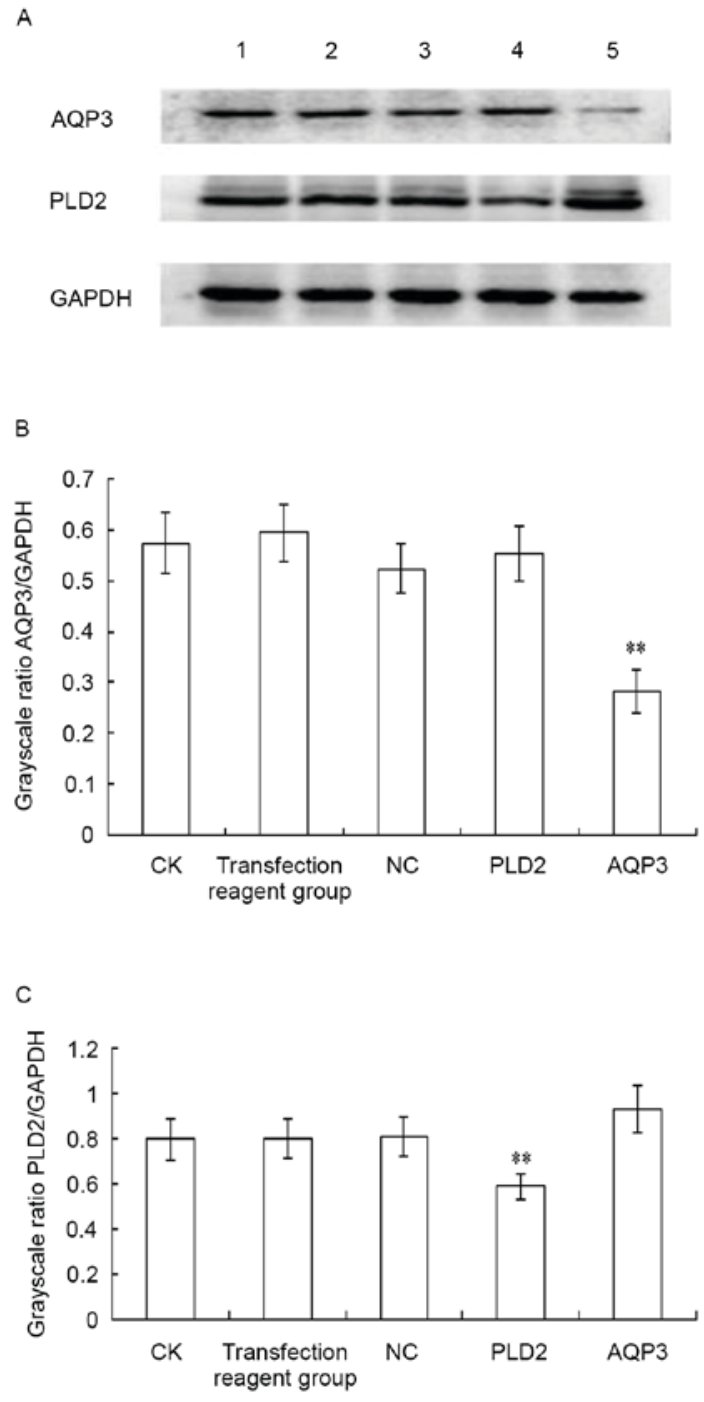

Figure 4. Inhibitory effect of siRNA transfection on the protein expression of AQP3 and PLD2. siRNA transfection and western blot analysis were performed. (A) Results of the western blot analysis; results are representative of three independent experiments. Quantification of the protein expression of (B) AQP3 and (C) PLD2. The statistical significance between two groups was determined using a paired-samples t-test. ${ }^{* *} \mathrm{P}<0.01$, compared with the NC group. Lane 1, CK group; lane 2, transfection reagent group (transfection reagent added to the culture medium); lane 3, NC group; lane 4, PLD2-siRNA group; lane 5, AQP3-siRNA group. siRNA, small interfering RNA; AQP3, aquaporin 3; PLD2, phospholipase D2; CK, control check; NC, negative control.

The suggestion that AQP3 and PLD2 are coupled is based on immunocytochemical co-localization and their ability to coimmunoprecipitate (12). The association of AQP3 and PLD2 in caveolin-rich membrane microdomains of keratinocytes and their functional association to generate the lipid signaling molecule PG provides a novel mechanism by which AQP3 regulates epidermal function. AQP3-mediated glycerol transport in skin is involved in a complex regulation of cell proliferation and differentiation, which are central features of epidermal homeostasis and regeneration. It is possible that AQP3 mediates different effects depending on whether or not it associated with PLD2 (26). In addition, manipulation of the AQP3/PLD2 signaling module appears to inhibit keratinocyte proliferation and trigger early differentiation (27). The 


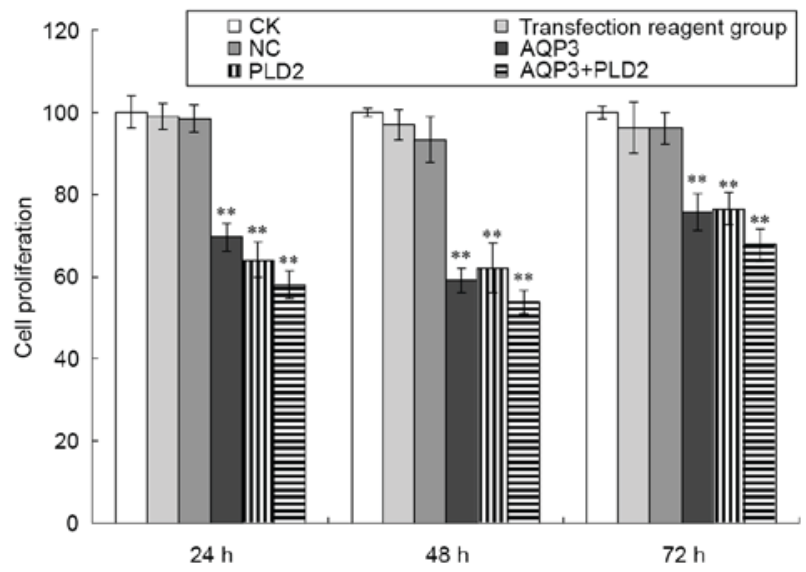

Figure 5. Inhibitory effect of siRNA transfection on the proliferation of A431 cells. Cell proliferation was analyzed and the results are representative of three independent experiments. The statistical significance between two groups was determined using a paired-samples t-test. ${ }^{* *} \mathrm{P}<0.01$, compared with the NC group. siRNA, small interfering RNA; AQP3, aquaporin 3; PLD2, phospholipase D2; CK, control check; NC, negative control.

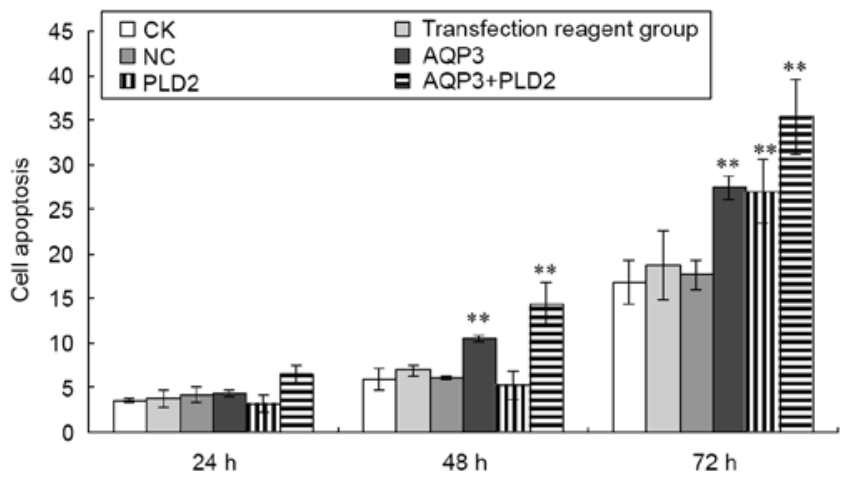

Figure 6. AQP3 siRNA and PLD2 siRNA increase the apoptosis of A431 cells. Cell apoptosis was determined; results are representative of three independent experiments. The statistical significance between two groups was determined using a paired-samples t-test. ${ }^{* *} \mathrm{P}<0.01$, compared with the NC group. siRNA, small interfering RNA; AQP3, aquaporin 3; PLD2, phospholipase D2; CK, control check; NC, negative control.

mechanism by which the AQP3/PLD2 signaling module exerts its effects on the pathogenesis of SCC remain to be fully elucidated, however, substantial data supporting an involvement of this signaling module in tumor progression and development indicate that further investigation is warranted.

AQP3 and PLD2 are abnormally expressed and/or localized in SCC. AQP3 transports glycerol to PLD2 for the synthesis of lipid second messengers, which potentially mediate the effects of the AQP3/PLD2 signaling module in regulating the proliferation and apoptosis of SCC.PLD exhibits cross talk with a variety of cancer regulators and also provides survival signals. There are multiple mechanisms by which PLD-mediated survival signals are generated in cancer cells. PLD suppresses phosphoprotein $2 \mathrm{~A}$, reduces its association with $\mathrm{E} 4 \mathrm{BP}$ and $\mathrm{S} 6 \mathrm{~K}$, and assists in the transformation of cells (28). PLD2 interacts with mammalian target of rapamycin and activates it, which provides survival signals (29). PLD stabilizes mutant p53 in a mitogen-activated protein kinase-dependent manner. In turn, PLD-generated survival signals dependent on mutant p53 (30). PLD also acts as a survival signal for cancer. PLD regulates hypoxia inducible factor $1 \alpha$ at the translational level and promotes cancer cell proliferation (29). Another mechanism by which PLD promotes cancer growth is by preventing the apoptosis of cancer cells. PLD2 promotes the survival of cancer cells by preventing apoptosis (31). PLD2 also enhances the expression of anti-apoptotic proteins, including B-cell lymphoma $2(\mathrm{Bcl}-2)$ and Bcl-extra large (32). To further elucidate the effective mechanism of AQP3 siRNA and PLD2 siRNA in the proliferation and apoptosis of $\mathrm{SCC}$, further investigation is necessary.

Taken together, the findings of the present study suggested that increased levels of AQP3 and PLD2 may be correlated with tumor progression and development in SCC. The results showed that AQP3 siRNA and PLD2 siRNA affected the proliferation and apoptosis of SCC. Transfection with AQP3 siRNA and PLD2 siRNA significantly inhibited the proliferation and promoted the apoptosis of SCC cells. The results also showed that AQP3 siRNA and PLD2 siRNA significantly downregulated the mRNA and protein levels of AQP3 and PLD2 in A431 cells, and that they inhibited proliferation and promoted apoptosis in vitro. The AQP3/PLD2 signaling module may be involved in, or serve as surrogate markers for the pathogenesis of SCC, and the concomitant downregulation of siRNA mediated by aberrant AQP3/PLD2 signaling may be important for the treatment of SCC. These findings provide novel insights for the development of gene therapy technology to treat patients with SCC in the future.

\section{Acknowledgements}

The authors would like to thank the staff of the Department of Pathology, Hangzhou Hospital of Traditional Chinese Medicine (Hangzhou, China), for their assistance with skin tissue sample collection and immunohistochemical analysis. This study was funded by the Natural Science Foundation of Zhejiang Province (grant no. LY12H11010).

\section{References}

1. Mobasheri A and Marples D: Expression of the AQP-1 water channel in normal human tissues: A semiquantitative study using tissue microarray technology. Am J Physiol Cell Physiol 286: C529-C537, 2004.

2. Boury-Jamot M, Sougrat R, Tailhardat M, Le Varlet B, Bonté F, Dumas M and Verbavatz JM: Expression and function of aquaporins in human skin: Is aquaporin-3 just a glycerol transporter? Biochim Biophys Acta 1758: 1034-1042, 2006.

3. Song Y, Sonawane N and Verkman AS: Localization of aquaporin-5 in sweat glands and functional analysis using knockout mice. J Physiol 541: 561-568, 2002.

4. Hara-Chikuma M, Sohara E, Rai T, Ikawa M, Okabe M, Sasaki S, Uchida S and Verkman AS: Progressive adipocyte hypertrophy in aquaporin-7-deficient mice: Adipocyte glycerol permeability as a novel regulator of fat accumulation. J Biol Chem 280: 15493-15496, 2005.

5. Boury-Jamot M, Daraspe J, Bonté F, Perrier E, Schnebert S, Dumas $\mathrm{M}$ and Verbavatz JM: Skin aquaporins: Function in hydration, wound healing, and skin epidermis homeostasis. Handb Exp Pharmacol 190: 205-217, 2009.

6. Sougrat R, Morand M, Gondran C, Barré P, Gobin R, Bonté F, Dumas $M$ and Verbavatz JM: Functional expression of AQP3 in human skin epidermis and reconstructed epidermis. J Invest Dermatol 118: 678-685, 2002.

7. Sugiyama Y, Ota Y, Hara M and Inoue S: Osmotic stress up-regulates aquaporin-3 gene expression in cultured human keratinocytes. Biochim Biophys Acta 1522: 82-88, 2001. 
8. Hara-Chikuma $M$ and Verkman AS: Prevention of skin tumorigenesis and impairment of epidermal cell proliferation by epidermal cell proliferation by targeted aquaporin-3 gene disruption. Mol Cell Biol 28: 326-332, 2008.

9. Liscovitch M, Czarny M, Fiucci G and Tang X: Phospholipase D: Molecular and cell biology of a novel gene family. Biochem J 3: 401-415, 2000

10. Liscovitch M, Chalifa V, Pertile P, Chen CS and Cantley LC: Novel function of phosphatidylinositol 4,5-bisphosphate as a cofactor for brain membrane phospholipase D. J Biol Chem 269: 21403-21406, 1994.

11. Gomez-Cambronero J: Phosphatidic acid, phospholipase D and tumorigenesis. Adv Biol Regul 54: 197-206, 2014.

12. Zheng X and Bollinger Bollag W: Aquaporin 3 colocates with phospholipase $\mathrm{d} 2$ in caveolin-rich membrane microdomains and is downregulated upon keratinocyte differentiation. J Invest Dermatol 121: 1487-1495, 2003.

13. Voss KE, Bollag RJ, Fussell N, By C, Sheehan DJ and Bollag WB: Abnormal aquaporin-3 protein expression in hyperproliferative skin disorders. Arch Dermatol Res 303: 591-600, 2011.

14. Ozdemir E, Kakehi Y, Okuno H and Yoshida O: Role of matrix metalloproteinase-9 in the basement membrane destruction of superficialurothelial carcinomas. J Urol 161: 1359-1363, 1999.

15. Wang XY, Tao CJ, Wu QY and Yuan CD: Protein extract of ultraviolet-irradiated human skin keratinocytes promote the expression of mitogen-activated protein kinases, nuclear factor- $\kappa \mathrm{B}$ and interferon regulatory factor-3 in Langerhans cells via Toll-like receptor 2 and 4. Photodermatol Photoimmunol Photomed 29: 41-48, 2013.

16. Livak KJ and Schmittgen TD: Analysis of relative gene expression data using real-time quantitative PCR and the 2(-Delta Delta C(T)) method. Methods 25: 402-408, 2001.

17. Wang X, Bi Z, Chu W and Wan Y: IL-1 receptor antagonist attenuates MAP kinase/AP-1 activation and MMP1 expression in UVA-irradiated human fibroblasts induced by culture medium from UVB-irradiated human skin keratinocytes. Int J Mol Med 16: 1117-1124, 2005.

18. Du G, Huang P, Liang BT and Frohman MA: Phospholipase D2 localizes to the plasma membrane and regulates angiotensin II receptor endocytosis. Mol Biol Cell 15: 1024-1030, 2004.

19. Verkman AS: A cautionary note on cosmetics containing ingredients that increase aquaporin-3 expression. Exp Dermatol 17 871-872, 2008

20. Majores M and Bierhoff E: Actinic keratosis, Bowen's disease, keratoacanthoma and squamous cell carcinoma of the skin. Pathologe 36: 16-29, 2015 (In German).
21. Min DS, Kwon TK, Park WS, Chang JS, Park SK, Ahn BH, Ryoo ZY, Lee YH, Lee YS, Rhie DJ, et al: Neoplastic transformation and tumorigenesis associated with overexpression of phospholipase D isozymes in cultured murine fibroblasts. Carcinogenesis 22: 1641-1647, 2001.

22. Min DS, Ahn BH and Jo YH: Differential tyrosine phosphorylation of phospholipase $\mathrm{D}$ isozymes by hydrogen peroxide and the epidermal growth factor in A431 epidermoid carcinoma cells. Mol Cells 11: 369-378, 2001.

23. Gomez-Cambronero J: New concepts in phospholipase D signaling in inflammation and cancer. ScientificWorldJournal 10: 1356-1369, 2010.

24. Holen T, Amarzguioui M, Wiiger MT, Babaie E and Prydz H: Positional effects of short interfering RNAs targeting the human coagulation trigger tissue factor. Nucleic Acids Res 30: 1757-1766, 2002.

25. Elbashir SM, Lendeckel W and Tuschl T: RNA interference is mediated by 21- and 22-nucleotide RNAs. Genes Dev 15: 188-200, 2001

26. Qin H, Zheng X, Zhong X, Shetty AK, Elias PM and Bollag WB Aquaporin-3 in keratinocytes and skin: Its role and interaction with phospholipase D2. Arch Biochem Biophys 508: 138-143, 2011.

27. Bollag WB, Xie D, Zhong X and Zheng X: A potential role for the phospholipase D2-aquaporin-3 signaling module in early keratinocyte differentiation: Production of a novel phosphatidylglycerol lipid signal. J Invest Dermatol 127: 2823-2831, 2007.

28. Hui L, Rodrik V, Pielak RM, Knirr S, Zheng Y and Foster DA: mTOR-dependent suppression of protein phosphatase $2 \mathrm{~A}$ is critical for phospholipase D survival signals in human breast cancer cells. J Biol Chem 280: 35829-35835, 2005.

29. Toschi A, Edelstein J, Rockwell P, Ohh M and Foster DA: HIF alpha expression in VHL-defficient renal cancer cells is dependent on phospholipase D. Oncogene 27: 2746-2753, 2008.

30. Hui L, Zheng Y, Yan Y, Bargonetti J and Foster DA: Mutant p53 in MDA-MB-231 breast cancer cells is stabilized by elevated phospholipase D activity and contributes to survival signals generated by phospholipase D. Oncogene 25: 7305-7310, 2006.

31. Cho JH, Hong SK, Kim EY, Park SY, Park CH, Kim JM, Kwon OJ, Kwon SJ, Lee KS and Han JS: Overexpression of phospholipase D suppresses taxotere-induced cell death in stomach cancer cells. Biochim Biophys Acta 1783: 912-923, 2008.

32. Oh KJ, Lee SC, Choi HJ, Oh DY, Kim SC, Min do S, Kim JM, Lee KS and Han JS: Role of phospholipase D2 in anti-apoptotic signaling through increased expressions of Bcl-2 and Bcl-xL. J Cell Biochem 101: 1409-1422, 2007. 